\title{
Haptic identification of objects and their depictions
}

\author{
ROBERTA L. KLATZKY and JACK M. LOOMIS \\ University of California, Santa Barbara, California \\ SUSAN J. LEDERMAN \\ Queen's University, Kingston, Ontario, Canada \\ HIROMI WAKE \\ Kanagawa University, Yokohama, Japan \\ and \\ NAOFUMI FUJITA \\ Kochi University, Kochi, Japan
}

\begin{abstract}
Haptic identification of real objects is superior to that of raised two-dimensional (2-D) depictions. Three explanations of real-object superiority were investigated: contribution of material information, contribution of 3-D shape and size, and greater potential for integration across the fingers. In Experiment 1, subjects, while wearing gloves that gently attenuated material information, haptically identified real objects that provided reduced cues to compliance, mass, and part motion. The gloves permitted exploration with free hand movement, a single outstretched finger, or five outstretched fingers. Performance decreased over these three conditions but was superior to identification of pictures of the same objects in all cases, indicating the contribution of 3-D structure and integration across the fingers. Picture performance was also better with five fingers than with one. In Experiment 2, the subjects wore open-fingered gloves, which provided them with material information. Consequently, the effect of type of exploration was substantially reduced but not eliminated. Material compensates somewhat for limited access to object structure but is not the primary basis for haptic object identification.
\end{abstract}

It has been amply demonstrated that people's ability to identify raised line drawings of common objects, using touch alone, is quite poor (Ikeda \& Uchikawa, 1978; Kennedy \& Fox, 1977; Lederman, Klatzky, Chataway, \& Summers, 1990; Loomis, Klatzky, \& Lederman, 1991; Magee \& Kennedy, 1980). Subjects may spend several minutes on a single picture, and accuracy is generally below 50\%-sometimes well below. This is especially striking when one considers that haptic identification of real, common objects is both fast and accurate, with modal response latency observed to be under 2 sec and accuracy near $100 \%$ (Klatzky, Lederman, \& Metzger, 1985). The purpose of the present study is to evaluate potential explanations of these differences in performance with real objects and two-dimensional (2-D) depictions. We consider three explanations, which are not meant to be mutually exclusive.

The first account of picture/object differences focuses on a salient aspect of haptic perception-namely, that the

We thank Andrew Beall and Judith Loftus for their assistance and acknowledge support from National Science Foundation Grant BNS8919383 and from the Centre of Excellence of the Ontario Information Technology Research Centre (ITRC). Requests for reprints may be sent to R. Klatzky, Department of Psychology, UCSB, Santa Barbara, CA $93106-9660$. "field of view" provided by the fingertip is quite restricted relative to vision. According to this account, objects provide a greater functional field of view than do pictures. A study of haptic picture identification by Loomis et al. (1991) indicated that performance was critically limited by field of view. Earlier studies by Becker (1935) and Yamane (1935) had displayed a visual pattern behind a small aperture, showing a similarity between vision and touch under these conditions. Loomis et al.'s more recent experiment attempted to equate the effective field of view for haptics and vision by constraining visual exposure to an aperture that was equivalent to the exploring fingerpad(s). In the haptic conditions, subjects explored raised drawings with either the index finger or the contiguous index and middle fingers. In the visual conditions, subjects explored a picture by moving an electronic pen over a digitizing tablet, the coordinates of which mapped onto the image stored in the computer memory. As the pen touched a location, the corresponding portion of the picture could be viewed within an aperture on a computer monitor. The size and shape of the aperture corresponded to the size and shape of one or two contiguous fingerpads, depending on the condition. When the visual aperture was stationary (hence the picture appeared to move behind it), the one-finger aperture size produced performance that was virtually identical to that obtained with touch-a mean accuracy of about $50 \%$ and response la- 
tencies averaging about $90 \mathrm{sec}$. Widening the aperture to two fingers had no effect on performance in the touch condition, but the increased aperture size did facilitate performance with vision. These results indicated, first, that a narrow field of view substantially limited picture perception in both modalities, and, second, that the modality of touch was unable to integrate information over the two adjacent fingers (see also Craig, 1985; Lappin \& Foulke, 1973), limiting the functional field of view beyond that defined by the physical aperture.

Exploration with a narrow field of view means that temporally separated samples of spatial contour must be integrated. People's limited ability to do the required integration is apparent in the low levels of performance and long RTs observed with sequential viewing by vision and touch. Widening the nominal field of view in touch, at least within the range of two fingers, appears not to widen the functional field, because two fingers function no better than one. Thus, the demands on integrative processing are apparently not reduced by using multiple fingers.

Considering these limitations, the low levels of haptic picture identification seem less surprising than does the success attained with identification of real objects. The account of these differences that we are considering proposes that the additional information provided by real objects offers greater potential for integration across the fingers. This seems particularly likely when exploration of real objects involves molding the hand to the object's contour. Kinesthetic information about the relative positions of finger and hand surfaces simultaneously contacting the object signals the layout of contour perhaps more effectively than scanning one finger across it. Size, for example, can be estimated by the gap between the opposing fingers that enclose an object (Chan, Carello, \& Turvey, 1990). Even when an object is explored with flat fingers, without molding the hand to its surface, a pressure gradient continued from one finger to another might be more effectively encoded than the segment underneath a single fingertip. In short, integrating information from different fingers is likely to be qualitatively different with real objects than it is with pictures. It could also be argued that greater integration occurs with real objects on a quantitative basis, assuming that they are more informative than pictures: When information is impoverished, as it is with raised tactile patterns (Loomis \& Lederman, 1986), compensatory processing resources may need to be expended in order to encode it, and the system may be taxed with the analysis of data from just a single finger. When the information is easily sensed, as with 3-D objects, it may be possible to spread processing capacity over a larger portion of the skin surface.

A second possible cause of real-object superiority in haptic identification is the potential use of material information when identifying real objects instead of pictures. A typical raised picture portrays only the object's shape in the form of an outer envelope and 2-D projections of internal edges. (Texture may also be portrayed by lines or stippling.) In contrast, a real object conveys haptically accessible infor- mation about the object's material, including surface roughness, compliance, and thermal properties such as conductivity. This difference may not be important in the domain of vision, in which shape appears to be the dominant cue to identification with either real objects or pictures. According to Biederman (1987), visual identification of an object requires the extraction of viewpoint-independent, primitive 3-D volumes, or geons. Relations among geons are then determined, with two to three geons and their arrangement being sufficient to determine an object's identity. That information about material properties is not required is indicated by the ease with which people visually identify line drawings, even when they are presented for only $100 \mathrm{msec}$ or so; in fact, no advantage is obtained from non-edgebased cues such as color (Biederman \& Ju, 1988). In the haptic domain, however, access to shape information is substantially reduced because of the limited field of view. The material cues available from real objects, but not from pictures, may then compensate.

A third possible reason for the discrepancy in haptic identification rates with pictures and objects is that the 3-D stimuli provide additional, haptically accessible structural (shape and size) information. Real objects have a fully 3-D volumetric structure and a meaningful absolute, as well as relative, size, which may be sensed sequentially or by the simultaneous enfolding of different regions of contour. There is added kinesthetic information, because the limb and finger positions needed to maintain contact vary in an additional dimension-namely, depth. Added cutaneous information results from meaningful pressure variations at the exploring fingertip. Pictures, in contrast, provide contour of uniform height, and thus it is solely the position of pressure points, rather than the magnitude of the pressure or its gradient, that is informative. Local cutaneous information is of little use except to mark the position of picture contour. Usually, no effort is made to keep a consistent mapping from the size of an object to the size of its depiction, so that relative size is not maintained across a set. (This was not the case in the present study, however, in which relative size was maintained.) Also, pictures are 2-D projections of 3-D objects. The perspective information must be interpreted to reconstruct the depicted depth, whereas with real objects, the third dimension is given directly.

The present experiments were performed to better elucidate the causes of differences in haptic recognition of pictures and objects. Much in the spirit of Katz (1989), we constrained the nature of the information available from 3-D objects and determined the effects on identification.

By identification, we mean naming at the "basic" level of categorization, at which objects are given the most agreed upon name and are most easily categorized (Rosch, Mervis, Gray, Johnson, \& Boyes-Braem, 1976). Objects from a common basic-level category tend to be highly similar in form and function, and those from distinct basiclevel categories are minimally confusable. Shape appears to be the most critical information for designating an object category at the basic level, whether the object is seen 
or touched (Lederman \& Klatzky, 1990; Rosch et al., 1976; Tversky \& Hemenway, 1984). Objects from a common basic-level category tend to share overall shape and part structure, so much so that a photographic average of several 2-D depictions (in prototypical perspective and normalized size) is recognizable (Rosch et al., 1976; Tversky \& Hemenway, 1984).

In Experiment 1, shape and size were virtually the only cues provided for haptic object identification. To reduce the potential contribution from nonstructural cues, the objects had no compliant parts, were fixed to a table (hence mass could not be assessed by lifting), and had any potentially movable parts (e.g., a detachable cover on a teapot) made rigid. The subjects wore gloves that also attenuated information about the object's material. To assess whether integration over the fingertips occurred, the objects were explored in one of three ways: with a single extended finger, with five extended fingers, and with the hand freely allowed to mold.

The subjects also attempted to name a set of raised pictures that were derived from photographs of the objects (their relative sizes were preserved). If it is the contribution of material information that underlies successful haptic object identification, we should expect to see performance in any of the haptic conditions descend close to the level of picture naming, because the gloves and fixed attachment of the stimuli greatly reduce this information. The third possible explanation for the discrepancy in haptic identification rates, which emphasizes the greater structural information provided by the 3-D stimuli, predicts that all three of the haptic conditions will be superior to picture naming. If there can be integration of kinesthetic information across the fingers with real objects, as proposed by the first explanation, mentioned above, allowing the hand to mold should produce superior performance compared with the use of a single finger, and there should be better performance with five extended fingers than with one.

Experiment 2 further assessed the contribution of material information to object naming. The subjects explored in the same ways as they did before, but now with their fingertips exposed to the object's surface, so that properties such as roughness and thermal conductivity became available. To the extent that material is important in identification, differences between exploratory conditions should be reduced. In particular, the contribution of material should increase as information about structure is increasingly limited.

\section{BASELINE PERFORMANCE}

In a preliminary study, we simply assessed the level of performance available in two full-cue conditions: when the objects were explored by touch (without vision) with an ungloved, unconstrained hand and when the twodimensional raised pictures were viewed.

\section{Materials}

The materials consisted of a set of 36 objects in real and pictorial form. The objects had no highly compliant parts, and any moving parts were glued to make them rigid. Each

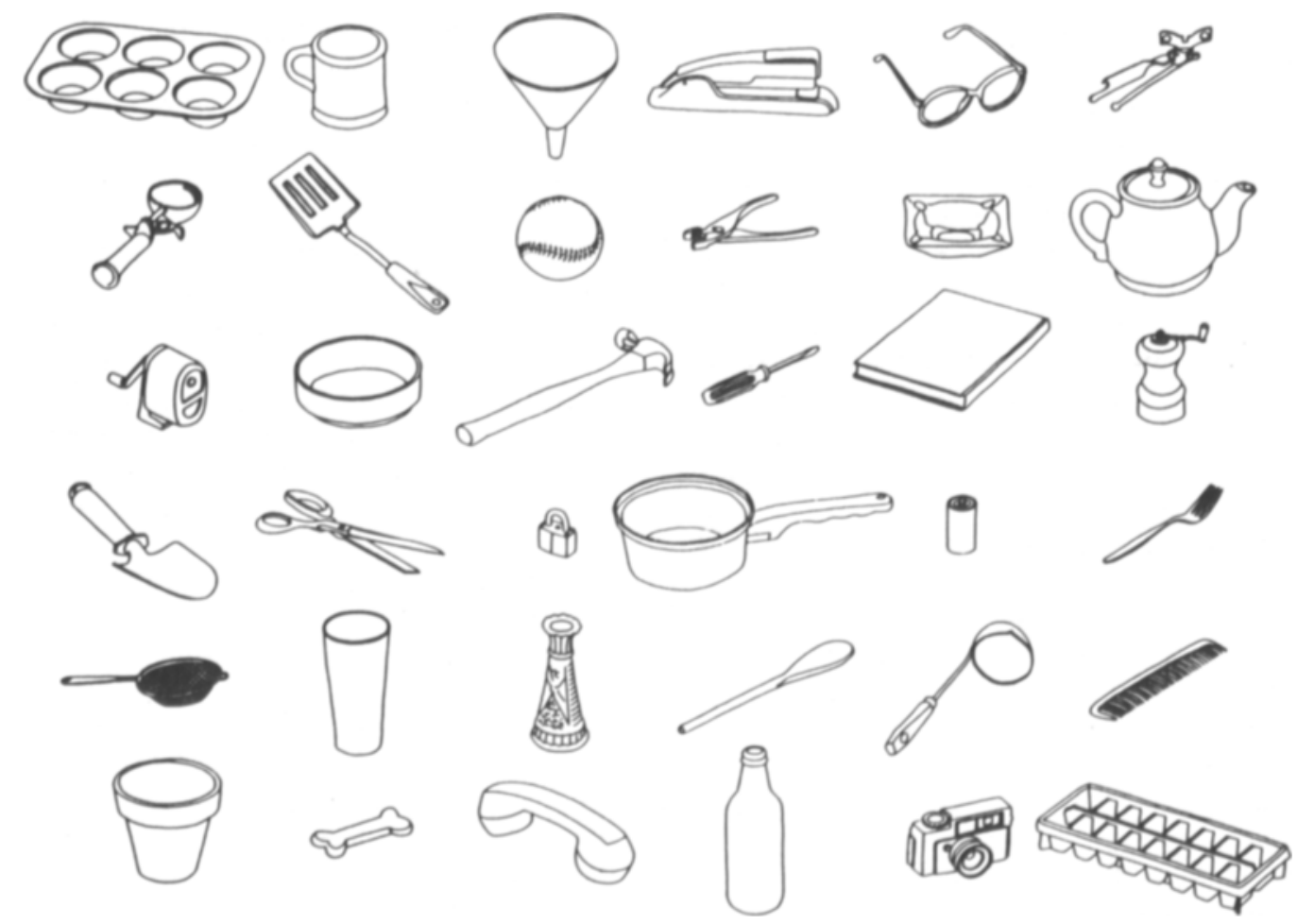

Figure 1. Picture stimuli used in Experiments 1 and 2, derived from photographs of the stimuli used in the realobject conditions. 
object was mounted on a Masonite board that was clamped to a table when the object was presented. The pictured counterparts were constructed by photographing the objects from a constant distance and elevation, then making a tracing of their essential features (i.e., excluding texture and luminance cues, but including internal lines representing edges). The drawings are shown in Figure 1. The tracing was then converted to a raised outline by xeroxing the drawings onto heat-sensitive paper that was put through a Matsumoto Stereo Copy Developer. The result was a raised picture with lines of uniform width and height $(.7 \times$ $.5 \mathrm{~mm}$ ), which have been shown to be highly legible under tactile exploration (Dacen \& Coulson, 1988). The still visible xeroxed lines in the raised pictures were of high contrast, with a stroke width of $.5 \mathrm{~mm}$. The depicted objects were slightly smaller than actual size (approximately a onethird reduction). Two additional real objects (nozzle and plate) and drawings (jar and candle) were used for practice trials, which preceded the experimental trials of that type.

\section{Procedure}

The subjects were 8 undergraduate students who were fulfilling a course requirement. Each subject was presented with the 36 distinct objects- 18 in the form of pictures (using vision) and 18 as real objects (using unrestricted touch). Across subjects, the order of the two presentation conditions was counterbalanced, and each object was tested in pictorial and in real form a total of four times. In the real-object condition, the subject was timed from the onset of contact until the response. In the picture condition, the subject was instructed to close the eyes between trials; he or she was timed by stopwatch from a signal to open the eyes up to the point of response.

\section{Results}

The results are simply the percentages of correct responses and the response times. Correct responses were allowed some leeway-for example, the mug could also be called a cup or a coffee cup. With the real objects, the mean RT was $6.12 \mathrm{sec}(S E M=1.02)$, and the accuracy averaged $95.1 \%(S E M=2.6)$. There was no item that was not recognized by at least 3 of the 4 subjects. With the pictures, mean RT was $1.28 \sec (S D=.22)$, and accuracy averaged $97.9 \%(S D=2.9)$. Only three items produced any errors (one per item): trowel, battery, and ashtray. Thus, accuracy in both baseline tasks was excellent, although the RT with real objects was substantially greater than the mean observed by Klatzky et al. (1985). The slower speed here is likely to reflect the reduction in cues from compliance, weight, and moving parts.

\section{EXPERIMENT 1}

As was described above, Experiment 1 compared picture and object identification under three types of exploration, which differed in the number of fingers used and-with real objects only-the ability to mold to the object's surface. Cues to the object's material were reduced because of the use of rigid objects fixed to a surface and the subject's wearing gloves.

\section{Method}

The subjects were 10 students at UCSB who were paid for their participation. The materials were as in the baseline study, except that only 35 objects were tested (the remaining object, the ashtray, was used as a practice item on real-object trials). The subjects were told that the pictures had been taken from an elevated position and were slightly smaller than their actual sizes.

Each subject took part in five conditions, three with real objects and two with pictures. In the real-object conditions, the subject wore a glove designed for winter wear. The fabric was knit covered with an outer smooth layer (approximately $3 \mathrm{~mm}$ thick when uncompressed); the palm and undersurfaces of the fingers (i.e., surfaces contacting the object) were further covered with a layer of vinyl (approximately $1 \mathrm{~mm}$ thick). To assess the extent to which material cues were available, two subjects, using a gloved fingertip and with the bare finger (rubbing was permitted), were asked to classify the stimulus objects (excluding two that were ambiguous) into one of seven classes of material. With the bare hand, performance averaged $58 \%$; it dropped to less than half as great with the glove (averaging $27 \%$, relative to $16 \%$ chance when adjusted for use of the responses).

In one condition (real-free), the glove was used without alteration, and the subject was allowed to explore freely. In a second condition (real-5), finger flexion was prevented by affixing a flat wooden splint measuring $11 \times 1 \mathrm{~cm}$ to the upper surface of each of the gloved fingers. The splint ran from the fingertip to just proximal to the metacarpophalangeal joint. Although it was possible to flex that joint, the subjects were instructed to keep the fingers flat and outstretched and not to grasp the object (contact with the palm was allowed); the experimenter monitored exploration for compliance with this instruction. In the third real-object condition (real-1), the subject was constrained to using the index finger only. A splint on that single finger restricted flexion, and the subject was told to keep the finger outstretched with the others curled into the palm. No gloves were worn in the two picture conditions. In one case (picture-5), the subject explored with all five fingers and palm, as desired. In the other (picture-1), only the index finger could be used. A 2-min limit on the response interval was imposed.

Each subject was tested with seven objects per condition; over subjects, all objects were tested equally in each condition. Two Latin squares were used to assign conditions to the subjects. There was one practice trial at the start of each condition.

\section{Results}

Figure 2 presents the mean RT and accuracy by condition, along with standard error bars. An analysis of variance (ANOVA) on the RT data with one five-level factor (i.e., excluding baseline conditions shown in the figure) showed a significant effect $[F(4,36)=32.3, p<.001]$. Contrasts testing differences between pairs of means, with alpha set at .05, showed that they were all significant except the picture-1/picture-5 comparison, which was marginal $(p<.10)$ and reached significance with a one-tailed $t$ test.

A corresponding ANOVA on the accuracy scores (again excluding baseline conditions) showed a significant main effect $[F(4,36)=40.22, p<.001]$. Contrasts showed differences between all pairs of means except real-5/real-free and real-1/real-free. The latter approached significance $(p<.10)$ and was significant with a one-tailed $t$ test. 

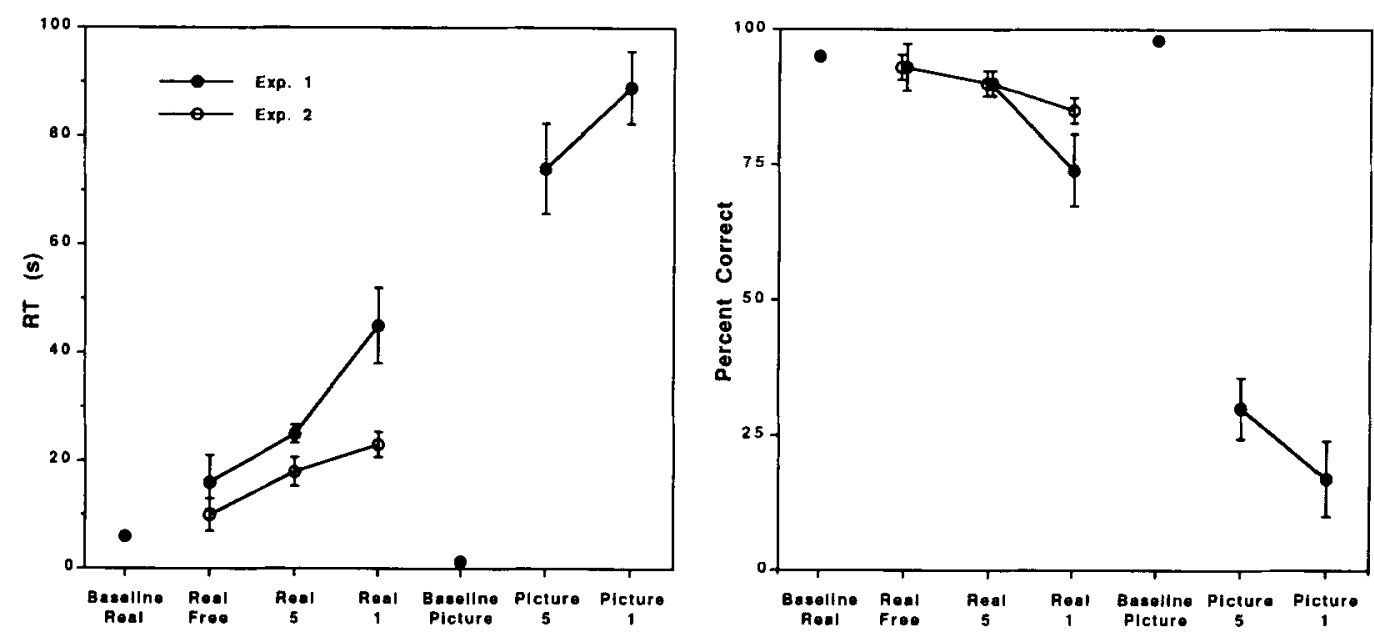

Figure 2. Mean RT (left panel) and accuracy (right panel) in the baseline conditions and the Experiments 1 and 2. The bars represent 1 SEM.

Correlations were computed across objects for each of the measures. Each object appeared in a given exploratory condition with two subjects. Their data were averaged, and the scores were then correlated between conditions over the set of $\mathbf{3 5}$ objects. The correlation matrix is shown in Table 1 . In general, RT and accuracy were negatively correlated, as expected. Intercorrelations among the realobject conditions were generally significant or nearly so, indicating common sources of variance. The picture-5 condition was also correlated to some extent with the realobject conditions. These correlations might reflect commonalities, at either perceptual levels (e.g., feature codability) or cognitive levels (e.g., name-access time). The failure to find significant between-condition correlations involving the picture- 1 condition does not seem to reflect lower variance caused by floor effects (see Figure 2 for standard errors); instead it may truly reflect distinct processes being applied to the task in this case.

\section{Discussion}

In general, the means differed (with respect to accuracy, $\mathrm{RT}$, or both) in accord with the idea that information about an object's shape is more haptically accessible with real objects than with pictures and the idea that integration oc- curs across the fingers with these stimuli. Although the present stimuli and exploratory constraints severely limited access to information about the object's material, real objects were still identified better than pictures. Allowing the hand to mold to the object enhanced performance relative to the condition in which the five fingers were held outstretched, which in turn was superior to exploration with a single finger, indicating integration across the fingers with real objects.

Performance with pictures was poor whether one or five fingers were used, relative to the baseline values for which pictures were viewed. This was expected from previous research. However, less expected was the finding that five fingers were better than one for picture identification. This contrasts with the results of Loomis et al. (1991), who found no advantage in haptic picture perception when two fingers were used rather than one. We will comment on this point further below.

Accuracy with real objects in the real-5 and real-free conditions was within $5 \%$ of mean accuracy in the baseline study, in which the subjects used the bare hand. However, neither condition showed a mean RT approaching the baseline value of $6.1 \mathrm{sec}$; in fact, none of the subjects in any real-object condition had a mean RT as low

Table 1

Correlations Across Objects for RT and Accuracy in Experiment 1, by Condition

\begin{tabular}{lrrrrrrrrr}
\hline & R1-RT & R1-AC & R5-RT & R5-AC & RF-RT & RF-AC & P1-RT & P1-AC & P5-RT \\
\hline R1-AC & -.67 & & & & & & & & \\
R5-RT & .36 & -.26 & & & & & & & \\
R5-AC & -.38 & .48 & -.66 & & & & & & \\
RF-RT & .50 & -.36 & .65 & -.43 & & & & & \\
RF-AC & -.29 & .17 & -.47 & .35 & -.38 & & & \\
P1-RT & .08 & -.09 & -.10 & .19 & .16 & .06 & & \\
P1-AC & .08 & .08 & -.13 & .15 & -.06 & -.04 & -.36 & & \\
P5-RT & .54 & -.44 & .14 & -.14 & .35 & -.21 & .18 & -.18 & -.58 \\
P5-AC & -.24 & .23 & -.37 & .28 & -.30 & .24 & .06 & .06 & - \\
\hline
\end{tabular}

Note-RT $=$ response time, $\mathrm{AC}=$ accuracy, $\mathrm{R} 1=$ real-1, $\mathrm{R5}=$ real-5, $\mathrm{RF}=$ real-free, $\mathrm{Pl}=$ picture-1, P5 $=$ picture-5. Correlations of .35 and above are significant at .05 . 
as $6.1 \mathrm{sec}$. Given that the procedural difference between the baseline condition and the real-free condition is whether the skin contacts the object's surface, the difference in performance between these conditions, albeit small, makes it clear that information provided by cutaneous sensing of surface properties contributes to identification of real objects. Experiment 2 further addressed this contribution.

\section{EXPERIMENT 2}

In the introduction, we considered the possibility that haptic recognition of objects differs from that of pictures because the former relies on material information that is not available from pictures. It could not be argued, however, that material is the exclusive basis for haptic object recognition, because shape information has been shown to be critical to object identity at the basic level. It is therefore questionable whether other information could compensate sufficiently to produce the speed and accuracy that have been observed in haptically based naming of real objects. However, material information could at least contribute to identification, if it did not constitute the exclusive basis.

Experiment 2 was intended to assess this contribution over the different exploratory conditions used in Experiment 1 . In this study, the subjects performed in the realobject recognition task wearing gloves that restricted movement, as in Experiment 1, but that allowed the skin of the fingertip to contact the object's surface. Thus, information was provided about material properties such as surface microstructure and thermal conductivity. If material information were the primary basis for haptic recognition of real objects, one would expect little difference between exploration with the free hand and with the extended fingers, although five extended fingers might still be superior to one. If material information were useful but did not override the contribution of structure, the exploration effects seen in Experiment 1 might be diminished but should still be present.

\section{Method}

The subjects were 12 students at UCSB who were fulfilling a course requirement; none had been in the previous studies. The stimulus set comprised the real objects used in the baseline study. Each subject took part in three conditions, corresponding to the real-1, real-5, and real-free conditions of Experiment 1, with 12 items per condition. Latin squares were used to assign the order of the conditions across subjects and to ensure that, over subjects, each stimulus was used in each condition equally often.

The subjects wore knit gloves with vinyl palms, similar to those worn in the preceding study (the approximate thicknesses of the knit fabric and vinyl were 3 and $1 \mathrm{~mm}$, respectively). However, in this case, the distal glove sections corresponding to the exploring fingers ( 1 or 5 , depending on condition) were cut off at a point determined by anthropometric norms for women to be just proximal to the distal interphalangeal joint, allowing contact between the object and the skin in that region. In the real-5 condition, $1 \mathrm{~cm}$ wide splints were applied to the undersurface of the thumb and four fingers, running from the palm area of the glove to the point where the glove terminated. (The undersurface was used because with the fingertips removed, the gloves could be pulled somewhat away from splints attached to the upper surface.) The subjects were told to keep the fingers flat, and their exploration was monitored by the experimenter. They were allowed to contact the object with the palm, although the termination of the splints there undoubtedly reduced information. In the real-1 condition, only the index finger was thus splinted. The subject was instructed to feel with that finger held flat and to flex the others toward the palm. In the real-free condition, exploration was unconstrained except for the use of the glove. Two practice trials using additional objects preceded a subject's first condition, using the gloves worn for that condition.

\section{Results and Discussion}

The RTs and accuracy scores are shown by condition in Figure 2. An ANOVA was run on the RTs $[F(2,22)=$ $16.14, p<.0001]$. Contrasts showed that the real-free condition was faster than either splinted condition; the difference between the two splinted conditions was significant only by a one-tailed $t$ test. A corresponding ANOVA was run on accuracy $[F(2,22)=5.34, p<.025]$. The difference between real-free and real-1 was significant when tested by a contrast, and the difference between real -1 and real- 5 was significant only by a one-tailed $t$ test. The real-5/real-free comparison did not approach significance.

The accuracy in the real-free condition, over $93 \%$, approached the baseline accuracy rate of $95 \%$. In fact, 5 of the 12 subjects had no errors with free exploration, and 3 subjects had no errors in the real- 5 condition. Thus, performance in these conditions is close to ceiling. In contrast, none of the subjects in the real-1 condition achieved $95 \%$ performance. The mean RT of $10.2 \mathrm{sec}$ in the freeexploration condition also approached the $6.1-\mathrm{sec}$ baseline value, with 4 of the 12 subjects showing RTs below $6 \mathrm{sec}$.

This experiment indicated that reduction of kinesthetic information affected performance even when cutaneous information about material was present. The use of a freely exploring hand led to better performance than when the hand was splinted so as to preclude enclosing the object; there was also a trend indicating that one (splinted) finger was inferior to five. Thus, access to material information cannot fully compensate for reduced information about shape.

Correlations between dependent variables in each condition were computed across the 36 objects, using the mean over the 4 subjects contributing to each object. These correlations are shown in Table 2 . The pattern is similar to that obtained with real objects in Experiment 1: RT and accuracy are negatively correlated, and there are reasonably strong correlations among the various conditions, which presumably reflect common perceptual and cognitive factors.

\section{Comparison Between Experiments}

The RTs in Experiment 2 were one-half to two-thirds as great as those in Experiment 1. Furthermore, the 3:1 ratio between the slowest and fastest conditions with real objects in Experiment 1 became only a doubling in Experiment 2. Given these differences, we compared the performance of the subjects in conditions common to Experiments 1 and 2 with an ANOVA that had type of exploration as one fac- 
Table 2

Correlations Across Objects for RT and Accuracy in Experiment 2, by Condition

\begin{tabular}{lrrrrr}
\hline & R1-RT & R1-AC & R5-RT & R5-AC & RF-RT \\
\hline R1-AC & -.50 & & & & \\
R5-RT & .52 & -.13 & & & \\
R5-AC & -.41 & .13 & -.73 & & \\
RF-RT & .50 & -.20 & .83 & -.65 & \\
RF-AC & -.46 & .32 & -.73 & .59 & -.92 \\
\hline
\end{tabular}

Note-RT $=$ response time, $A C=$ accuracy, $R 1=$ real $-1, \mathrm{R} 5=$ real-5, $\mathrm{RF}=$ real-free. Correlations of .32 and above are significant at the .05 level.

tor (real-1, real-5, and real-free) and fingertip status (covered, Experiment 1; uncovered, Experiment 2) as another. (Although the subjects were not randomly assigned to the two experiments from a common pool, the populations were very similar.) All three effects were significant in the RT analysis: for exploration $[F(2,40)=24.60, p<$ $.0001]$, for fingertip status $[F(1,20)=12.09, p<.01]$, and for the interaction $[F(2,40)=4.88, p<.05]$. Thus, the analysis confirmed that the open glove led to faster RT overall and reduced the effects of the exploratory condition.

Accuracy scores in the two experiments were very similar; in fact, they were virtually identical for the real-free and real -5 conditions. Hence, the corresponding analysis on accuracy scores showed only effects of exploration $[F(2,40)=7.81, p<.01]$.

The experiments allowed essentially equal access to structural information-what distinguishes them is access to material. The relative difficulty of the various objects would be expected to differ most, then, in the conditions in which material can substantially contribute to recognition in one study but not in the other. In particular, performance is likely to differ between the two studies when structural information is most restricted-that is, when subjects explore with a single extended finger. This can be seen by comparing the means between experiments in Figure 2.

The correlations between the two experiments are in further agreement with this reasoning. They are shown for corresponding conditions in Table 3 . (Correlations are over the 35 objects common to both experiments.) These correlations indicate whether objects that were found to be relatively difficult or easy in one experiment were similarly difficult or easy in the other. They tend to be lower, the less easily the subject can obtain structural information about the object. This is most evident with respect

Table 3

Correlations between Experiments 1 and 2

With Respect to Measures on Real Objects

\begin{tabular}{lc}
\hline Measure & Correlation \\
\hline R1-RT & .35 \\
R5-RT & .53 \\
RF-RT & .80 \\
R1-AC & .19 \\
R5-AC & .52 \\
RF-AC & .39 \\
\hline
\end{tabular}

Note- $\mathrm{RT}=$ response time, $\mathrm{AC}=$ accuracy, $\mathbf{R} 1=$ real $-1, \mathbf{R 5}=$ real-5, $\mathrm{RF}=$ real-free. Correlations of .32 and above are significant. to RTs, but, for accuracy as well as for RT, the correlation is lowest in the real-1 condition, in which subjects should make the greatest use of information about the object's material when available, due to the restrictions on encoding its structural properties.

We conducted a further test of the idea that the results of the two experiments would differ most for conditions in which structural information was most limited; therefore, material could make a substantial contribution in Experiment 2 (but not in Experiment 1). A factor analysis was performed on the basis of the correlations within and between Experiments 1 and 2 with respect to real-object performance (which use objects as the units of observation). Table 4 shows the solution with orthogonal rotation, which indicated three underlying factors accounting for $72 \%$ of the variance. The collection of variables with high loadings on a given factor can be interpreted as representing some underlying determinant of performance.

The analysis suggested that different aspects of the stimuli contributed to performance in the one-finger exploration conditions in Experiments 1 and 2 and that these conditions were distinct from the conditions with five fingers. The first factor, which received high loadings from all of the five-finger conditions in both experiments, is likely to represent the influence of shape information, which would be highly available for those conditions. The separation of the next factor, with loadings from the one-finger conditions in Experiment 1, indicates that performance in those conditions differed from that with five fingers even when access to material was restricted. Given the constraints of Experiment 1, this factor seems likely to reflect the use of shape information, but information that was apparently of a different sort than that available in the five-finger conditions. The last factor, representing one-finger performance in Experiment 2, presumably indicates reliance on material properties, given impoverished access to shape.

One might wonder whether performance in the real-1 condition in either experiment was correlated with picture performance in Experiment 1, since these conditions all show low correlations with the other real-object measures. However, the correlations between real-1 and picture measures were generally low. This can be seen for Experiment 1 by examination of Table 1 . The real-1 RT and accuracy measures in Experiment 2 showed similarly low correlations with the four picture measures of Experiment 1 ; only one of the eight $r$ values reached significance, and then narrowly (a value of .34 between real-1 RT in Experiment 2 and picture-5 RT in Experiment 1). 
Table 4

Factor Loadings for Measures in Experiments 1 and 2, Based on the Correlations Across Objects

\begin{tabular}{|c|c|c|c|c|}
\hline & Factor 1 & Factor 2 & Factor 3 & \\
\hline \multicolumn{5}{|l|}{ Real-1 Condition } \\
\hline $\begin{array}{l}\text { Experiment } 1: \mathrm{RT} \\
\text { Experiment } 1: \mathrm{AC} \\
\text { Experiment } 2: \mathrm{RT} \\
\text { Experiment } 2: \mathrm{AC}\end{array}$ & $\begin{array}{r}-0.298 \\
0.120 \\
-0.401 \\
-0.065\end{array}$ & $\begin{array}{c}0.858^{*} \\
-0.875^{*} \\
0.183 \\
0.054\end{array}$ & $\begin{array}{c}0.109 \\
0.197 \\
-0.615^{*} \\
0.933^{*}\end{array}$ & \\
\hline \multicolumn{5}{|l|}{ Real-5 Condition } \\
\hline $\begin{array}{l}\text { Experiment 2: RT } \\
\text { Experiment } 2: \mathrm{AC} \\
\text { Experiment } 1: \mathrm{RT} \\
\text { Experiment } 1: \mathrm{AC}\end{array}$ & $\begin{array}{r}-0.675^{*} \\
0.813^{*} \\
-0.724^{*} \\
0.432^{*}\end{array}$ & $\begin{array}{r}0.469 \\
-0.279 \\
0.170 \\
-0.361\end{array}$ & $\begin{array}{r}-0.216 \\
0.070 \\
-0.245 \\
0.406\end{array}$ & \\
\hline \multicolumn{5}{|l|}{ Real-free Condition } \\
\hline $\begin{array}{l}\text { Experiment } 1: \mathrm{RT} \\
\text { Experiment } 1: \mathrm{AC} \\
\text { Experiment } 2: \mathrm{RT} \\
\text { Experiment } 2: \mathrm{AC}\end{array}$ & $\begin{array}{r}-0.658^{*} \\
0.861^{*} \\
-0.712^{*} \\
0.632^{*}\end{array}$ & $\begin{array}{r}0.376 \\
0.026 \\
0.413 \\
-0.410\end{array}$ & $\begin{array}{r}-0.289 \\
-0.159 \\
-0.363 \\
0.463\end{array}$ & \\
\hline Variance Explained: & $34.8 \%$ & $20.6 \%$ & $16.9 \%$ & Total: $72.4 \%$ \\
\hline
\end{tabular}

\section{GENERAL DISCUSSION}

The present findings illuminate a number of related issues concerning the haptic identification of real objects and its relation to that of raised, 2-D depictions. One issue is simply the comparability of these two types of tasks when items are matched in name and relative size. A second issue, which is the principal focus of this paper, concerns the underlying causes of observed differences in identification rates and latencies, which can be addressed by observing how identification performance varies with the method of exploration.

With respect to the first of these issues, our results with matched stimuli confirm that although people can recognize real objects by touch quite well, their performance with raised pictures of those same objects is remarkably poor. The present success rate for pictures explored with five fingers $(30 \%)$ is similar to the $34 \%$ obtained by Lederman et al. (1990) and somewhat below the $45 \%$ found by Loomis et al. (1991); the response latencies in all three studies are roughly comparable.

Some aspects of the present study might lead to overestimation of the difference between haptic picture and object recognition. One is the prevalence of 3-D information in the present drawings, since the stimuli were depicted from an elevated viewpoint. Three-dimensional cues have sometimes been found to deter haptic picture recognition (Lederman et al., 1990), but not consistently (Loomis et al., 1991). Another possible factor is the size reduction of the stimuli, although the typical picture pool in such studies deprives subjects of both relative and absolute size, whereas relative size was maintained here. Restriction of the means of exploration may have reduced performance. Heller (1989) observed that late-blind subjects tended to use both hands to explore pictures and outperformed sighted subjects who used a single finger. It might also be argued that subjects have limited experience with the task of haptic picture recognition, an argument that is not specific to the present study. Although experience could indeed be a factor and training might improve performance, one should note that experience with identifying real, 3-D objects by restricted means of touching is also likely to be very limited. Unfamiliarity with the task is therefore common to the pictorial and real-object conditions. Also common to those conditions are a number of other factors that might affect performance, such as prior familiarity with the object or frequency of use of its name; thus these factors alone cannot account for the low level of picture identification.

Despite the considerable difference in absolute performance levels, correlations between performance measures from the real-object and picture conditions suggest that the relative difficulty of an individual item is similar to some extent, whether it is presented as a real object or a raised picture. The common sources of variance may reflect shared higher-order components of the task, such as retrieving the object's name once its category is known. However, it is also possible that processes are shared at lower levels.

In the introduction, we considered three potential explanations of the real-object superiority effect in haptic identification: greater integration across the fingers with real objects, use of material information in identification of real objects but not pictures, and contribution of 3-D shape and size with real objects. We stated at the outset that these three accounts were not mutually exclusive, and indeed, evidence for all three mechanisms was obtained from the present studies.

The effects of exploratory constraints on real objects indicate that performance was facilitated both by a greater number of exploring fingers and by the ability to grasp the objects. These results are consistent with the ideas that the 
3-D variations in real-object contours are highly informative and promote integration across the fingers. With real objects, unlike with raised pictures, the gradient of pressure, as well as its spatial location, is likely to be an effective cue. Relatively good performance when exploring with a single outstretched finger attests to the effectiveness of this additional information. The differences between one and five outstretched fingers further indicate that integration of cutaneous and/or kinesthetic information occurs, and the additional advantage from free molding with the hand indicates a contribution of kinesthetic information about simultaneously contacted 3-D contour.

There were also indications of integration with 2-D displays. In contrast to our previous results (Loomis et al., 1991), performance was slightly better when pictures were felt with a greater hand surface, or "aperture." This might be attributed to two differences in procedure: The aperture manipulation here was more extreme (one vs. five fingers; cf. one vs. two fingers in the previous study), and the present subjects were not required to hold the fingers together. As a result, the spatial extent of simultaneously sampled contour would be considerably greater in the multiple-finger condition of this study than it was previously. It is also possible that multiple fingers play a role in guiding exploration, rather than perceptual integration in the form of a widened effective field of view.

The high levels of performance achieved in Experiment 1 , in which the hands were gloved and cues to material were strongly reduced, indicate that real objects can successfully be recognized by structural cues (shape and size). However, response latencies were slower than those observed with an uncovered hand. The comparison between the two experiments, in which the subjects had the fingertips either covered (Experiment 1) or exposed (Experiment 2), provides further understanding of the contribution of an object's material to its identification. The results suggest that when subjects have access to ample information about structure, from their use of the full hand with or without grasping, additional cutaneous information about material produces only a small facilitation. When structural information is limited to what can be obtained from a single exploring digit, the addition of material information has a more substantial effect on object identification, although still not enough to bring the singlefinger condition to the level of speed or accuracy obtained with the full hand.

Correlational data support the assumption that information about material contributes to identifying an object more in the single-finger condition than in the other exploratory conditions. A factor analysis based on correlation across objects indicated that there were three factors underlying the various real-object performance measures in the two experiments, with distinct factors for the multiple- and the single-finger conditions, with and without material information available. We have tentatively suggested that the first of these factors represents shape information derived from simultaneous contributions from multiple fingers, whereas the second factor represents contours extracted by a single finger and the third factor corresponds to the contribution of material. Although we cannot be certain about these attributions, the factor analysis clearly indicates that the information used to identify an object from single- and multiple-finger exploration differs not only when information about material is present but also when it is greatly attenuated.

\section{REFERENCES}

Becker, J. (1935). Über taktilmotorische Figurwahrnehmung. Psychologische Forschungen, 20, 102-158.

Biederman, I. (1987). Recognition-by-components: A theory of human image understanding. Psychological Review, 94, 115-147.

Biederman, I., \& Ju, G. (1988). Surface versus edge-based determinants of visual recognition. Cognitive Psychology, 20, 38-64.

Chan, T.-C., Carello, C., \& Turvey, M. T. (1990). Perceiving object width by grasping. Ecological Psychology, 2, 1-35.

Craig, J. C. (1985). Attending to two fingers: Two hands are better than one. Perception \& Psychophysics, 38, 496-511.

DaCen, D., Coulson, M. (1988). Tactile mobility maps-A comparative study. In A. F. Tatham \& A. G. Dodds (Eds.), Proceedings of the Second International Symposium on Maps and Graphics for Visually Handicapped People (pp. 7-23). Nottingham: University of Nottingham Press.

Heller, M. A. (1989). Picture and pattern perception in the sighted and the blind: The advantage of the late blind. Perception, 18, 379-389.

IKEDA, M., \& UCHIKAWA, K. (1978). Integrating time for visual pattern perception and a comparison with the tactile mode. Vision Research, 18, 1565-1571.

KATZ, D. (1989). The world of touch (L. E. Knueger, Ed. and Trans.). Hillsdale, NJ: Erlbaum.

Kennedy, J. M., \& Fox, N. (1977). Pictures to see and pictures to touch. In D. Perkins \& B. Leondar (Eds.), The arts and cognition (pp. 118-135). Baltimore: Johns Hopkins University Press

Klatzky, R. L., Lederman, S. J., \& Metzger, V. A. (1985). Identifying objects by touch: An "expert system." Perception \& Psychophysics, 37, 299-302.

LAPPIN, J. S., \& FoulKE, E. (1973). Expanding the tactual field of view. Perception \& Psychophysics, 14, 237-241.

Lederman, S. J., \& KLATZKy, R. L. (1990). Haptic classification of common objects: Knowledge-driven exploration. Cognitive Psychology, 22, 421-459.

Lederman, S. J., Klatzky, R. L., Chataway, C., \& Summers, C. D. (1990). Visual mediation and the haptic recognition of twodimensional pictures of common objects. Perception \& Psychophysics, 47, 54-64.

Loomis, J., KlatzKy, R. L., \& Lederman, S. J. (1991). Similarity of tactual and visual picture recognition with limited field of view. Perception, 20, 167-177.

Loomis, J., Lederman, S. (1986). Tactual perception. In K. Boff, L. Kaufman, \& J. Thomas (Eds.), Handbook of human perception and performance (pp. 1-41). New York: Wiley.

MAGEE, L. E., \& KENNEDY, J. M. (1980). Exploring pictures tactually. Nature, 278, 287-288.

Rosch, E., Mervis, C., Gray, W., Johnson, D., \& Boyes-Braem, P. (1976). Basic objects in natural categories. Cognitive Psychology, 8 , 382-439.

TVERSKY, B., \& HEMENWAY, K. (1984). Objects, parts, and categories. Journal of Experimental Psychology: General, 113, 169-193.

YAMANE, K. (1935). Tactile pattern perceptions [in Japanese]. Japanese Journal of Psychology, 10, 327-390.

(Manuscript received June 8, 1992; revision accepted for publication January 13, 1993.) 